\title{
Efecto de la incorporación de transglutaminasa microbiana en las propiedades sensoriales de hamburguesas de desmenuzado de merluza (Merluccius hubbsi)
}

\author{
Influence of microbiological transglutaminase on the sensory \\ properties of burgers of minced hake (Merluccius hubbsi)
}

\begin{abstract}
$\underline{\text { Panuncio, Alicia }}^{(1)}$, Cardeza, Luciana ${ }^{(1)}$, Quintero, Mariana ${ }^{(1)}$, Solé, María Laura ${ }^{(1)}$, Barrios, Sofía $^{(2)}$, Gámbaro, Adriana $^{(3)}$
(1) Instituto de Investigaciones Pesqueras, Facultad de Veterinaria, UdelaR, Uruguay - (2) Departamento de reactores, Instituto de Ingeniería Química, Facultad de Ingeniería, UdelaR, Uruguay - ${ }^{(3)}$ Sección Evaluación Sensorial, Departamento de Alimentos, Facultad de Química, UdelaR, Uruguay.
\end{abstract}

Contacto: alpanu08@gmail.com

Recibido: 17/6/2013 - Aprobado: 24/10/2013

\begin{abstract}
$\underline{\text { Resumen }}$
Se elaboraron hamburguesas de pescado a partir de desmenuzado de merluza (Merluccius hubbsi) con la incorporación de transglutaminasa microbiana, con la finalidad de mejorar sus propiedades sensoriales y elaborar un producto pesquero que fomente el consumo de pescado en Uruguay. Se evaluó desde el punto de vista sensorial y de textura instrumental el efecto del agregado de la enzima en la formulación de las hamburguesas. Se elaboraron tres tipos de hamburguesas: sin enzima y con incorporación de $0,5 \%$ y $1,0 \%$ de enzima transglutaminasa, respectivamente. Para la evaluación sensorial se analizaron las muestras con un panel de seis jueces entrenados y para la determinación de los parámetros de textura instrumental se utilizó un analizador de textura TA.XT2i. Los resultados de ambos estudios indicaron que el agregado de enzima mejoró sustancialmente la apariencia, la textura y el sabor del producto. Se obtuvo un producto pesquero innovador, con características que pueden resultar muy atractivas para el consumidor uruguayo.

Palabras clave: Hamburguesas, merluza desmenuzada, transglutaminasa, evaluación sensorial.
\end{abstract}

\begin{abstract}
Burgers of minced hake (Merluccius hubbsi) were prepared with the addition of microbial transglutaminase, in order to improve sensorial properties and hence to develop a fishery product that encourage the consumption of fish in Uruguay. The effect of the enzyme was evaluated by sensory and instrumental analysis. Three types of hamburgers were prepared: two with transglutaminase $(0,5 \%$ and $1,0 \%)$ and a control sample without enzyme. A panel of six trained sensory assessors identified the most relevant descriptors and provided the corresponding ratings on an individual basis. Instrumental texture measurements were performed with a texture analyzer TA.XT2i. The obtained results suggest that the addition of microbial transglutaminase substantially improved the appearance, texture and flavor of the product. An innovating fishing product was obtained with features that can be very attractive to Uruguayan consumers.

Keywords: Burgers, minced hake, transglutaminase, sensorial evaluation.
\end{abstract}

\section{Introducción}

La venta de pescado anual en Uruguay se puede estimar en $1.904 .900 \mathrm{~kg}$. En Montevideo, el consumo por habitante tuvo un incremento de 9,1 kg en 1997 a 12,6 kg en 2007 (López, 2007). Comparativamente, España tiene un consumo de $39 \mathrm{~kg}$ y Japón alcanza $98 \mathrm{~kg}$ por habitante por año. La principal forma de presentación de los productos es el filete, y es la forma de comercialización de aproximadamente el $75 \%$ del pescado en las ferias. Según los propios vendedores, la venta de pescado entero ha decaído, suplantado por los filetes, una alternativa más cómoda para el consumidor uruguayo. No se evidencia un aumento en la cantidad de pescado consumida en los últimos años, aunque recientemente el consumo de pescado azul ha ido en aumento por las indicaciones médicas de las bondades de los ácidos grasos omega 3 (López, 2007).
La merluza (Merluccius hubbsi) es una de las principales especies vendidas en nuestro país. Presentada en lomos, su comercialización en los diferentes puntos de venta varía según la disponibilidad. Otra forma en la que se la encuentra en el mercado nacional es el desmenuzado (o "minced", como se conoce en la industria), una masa compacta rectangular de 7,5 kg de pescado picado, congelada en armario de placas múltiples. Se obtiene principalmente luego de realizado el fileteado y se utiliza la porción que se encuentra adosada a la columna vertebral, recortes y tamaños de filetes de talla inadecuados para otros fines que se colocan en la máquina extractora de pulpa. De este modo se recupera pulpa para consumo humano directo, dado que generalmente en las plantas pesqueras esta porción se envía para hacer harina de pescado para consumo animal. Otra posibilidad es que el desmenuzado se obtenga luego de realizado el corte espalmado o mariposa a la merluza y ulteriormente procesado en la despulpadora. 
Debido al bajo consumo de pescado en Uruguay (Mazza Pérez, 2000) se consideró el desarrollo de una hamburguesa de desmenuzado de merluza como una forma más atractiva y económica de consumir pescado, y se optó por el estudio de la aplicación de la enzima transglutaminasa para mejorar sus propiedades sensoriales.

La transglutaminasa (TG) se encuentra en los tejidos animales y vegetales y en los fluidos corporales de los seres vivos. Al principio se conocía solamente la enzima de origen animal, pero la cantidad que se extraía era escasa, de mediana calidad y requería de calcio como cofactor. En 1994 se descubrió la especie de microorganismo más eficiente en la producción de esta enzima, Streptoverticillium mobaraense, con actividad independiente del calcio (Sakamoto et al., 1994). La TG es una enzima capaz de unir proteínas entre un grupo $\varepsilon$-amino de un residuo de lisina y un grupo carboxiamida de un residuo de glutamina, mediante un enlace covalente. Por ende, se genera un enlace inter o intramolecular muy resistente a la proteólisis. La TG microbiana se utiliza en diversos productos alimenticios tales como carne de bovino y de ave, productos panificados, quesos, etcétera (Aguilar-Zárate, 2012; Márquez et al., 2006; Steffolani, 2012).

El empleo de transglutaminasa mejora la formación de geles de pescado. La TG ha sido empleada para obtener surimi de jurel (Trachurus murphyi) y abadejo de Alaska (Theragra chalcogramma) (Asagami et al., 1995; Dondero et al., 2002; Gilleland et al., 1997). Asagami et al. (1995) adicionaron TG en el surimi congelado de diferentes especies demostrando que los efectos de la TG no solo dependían de la especie de la que se extrae el músculo, sino también de otros factores, como por ejemplo su frescura.

El objetivo de este trabajo fue estudiar la influencia de la incorporación de transglutaminasa microbiana en la textura y el sabor de hamburguesas de desmenuzado de merluza.

\section{Materiales y Métodos}

Se utilizó como materia prima desmenuzado de merluza (Fripur S.A.) y transglutaminasa microbiana (Eresur S.A./Ajinomoto). Se elaboraron tres embutidos de $500 \mathrm{~g}$ cada uno, a los que se les incorporó distintas concentraciones de TG $(0,5 \%$ de enzima, $1,0 \%$ de enzima, según recomendación del proveedor, y un blanco sin enzima). Para preparar cada uno de los embutidos se pesaron $500 \mathrm{~g}$ de merluza desmenuzada, se colocaron en un recipiente de acero inoxidable y se le agregó la cantidad de enzima especificada, previamente pesada y mezclada con agua en una relación 1:6. La temperatura de trabajo fue de $20 \pm 2{ }^{\circ} \mathrm{C}$. Las preparaciones se realizaron por triplicado. Las formulaciones se observan en la Tabla 1.

\begin{tabular}{|c|c|c|c|}
\hline & Sin enzima & $\mathbf{0 , 5 \%}$ de enzima & $\mathbf{1 , 0} \%$ de enzima \\
\hline Pescado & $500 \mathrm{~g}$ & $500 \mathrm{~g}$ & $500 \mathrm{~g}$ \\
\hline Enzima & $0 \mathrm{~g}$ & $2,5 \mathrm{~g}$ & $5 \mathrm{~g}$ \\
\hline Agua & $0 \mathrm{~g}$ & $15 \mathrm{~mL}$ & $30 \mathrm{~mL}$ \\
\hline \hline
\end{tabular}

Tabla 1. Formulaciones de cada tipo de hamburguesa.

La preparación de TG declarada por el proveedor está compuesta por: cloruro de sodio, gelatina, fosfato trisódico, maltodextrina, transglutaminasa y aceite de cártamo. El pH de la preparación es 11 .
Luego se mezcló el pescado y el preparado de enzima durante cuatro minutos para homogenizar bien la muestra. Pasado ese tiempo se colocó sobre un film adherente de PVC y se le dio la forma de un embutido de 5,4 cm de diámetro y $21 \mathrm{~cm}$ de largo. Para que su forma quedara homogénea se pasó por un anillo de acero inoxidable de 5,4 $\mathrm{cm}$ de diámetro, y de esta manera se aseguró la homogeneidad en la forma de las hamburguesas, facilitando así una cocción pareja.

Las distintas muestras se almacenaron por separado en envases plásticos y en heladera a $4{ }^{\circ} \mathrm{C}$ durante 20 horas. Posteriormente se cortaron los embutidos, obteniendo hamburguesas de $1 \mathrm{~cm}$ de espesor.

Para la cocción de las muestras se utilizó un horno eléctrico (Punktal, modelo PK-120A) previamente calentado. La temperatura de cocción utilizada fue de $240{ }^{\circ} \mathrm{C}$ y se cocinó cada hamburguesa durante 7 minutos y medio de cada lado. Debajo de cada unidad se colocaron cinco gotas de aceite de girasol.

\section{Evaluación sensorial}

La evaluación sensorial fue llevada a cabo por seis integrantes del panel de jueces de Facultad de Química, seleccionados y entrenados según normativa ISO 8586-1 (1993) y con un mínimo de 200 horas de experiencia en pruebas discriminativas y descriptivas de distintos tipos de alimentos.

Para generar los descriptores se cocinaron tres hamburguesas de cada tipo y se les presentaron juntas a los jueces. Primero, los jueces debieron generar los descriptores de manera individual y luego, en una discusión abierta con el líder de panel, se definieron por consenso los descriptores definitivos y su forma de evaluación. Los descriptores seleccionados fueron: forma, aspecto, color, dureza (fuerza requerida para comprimir una sustancia con los dientes molares o con la lengua y el paladar), humedad bucal, masticabilidad (número de veces que hay que masticar el alimento para poder tragarlo), sabor salado e intensidad total de sabor.

Para su evaluación las muestras fueron presentadas a los jueces en orden balanceado y codificadas con tres números al azar, junto con la boleta de evaluación correspondiente. Se utilizó agua sin gas como borrador. Los jueces evaluaron la intensidad de los descriptores seleccionados con una escala no estructurada de $10 \mathrm{~cm}$. Los extremos de las escalas asignados a cada descriptor se observan en la Tabla 2. Las evaluaciones se realizaron por duplicado en un laboratorio de evaluación sensorial que fue diseñado según la norma ISO 8589 (1988).

Sobre los datos obtenidos se realizó un análisis de varianza (ANOVA) utilizando "juez", "repetición”, "muestra” y sus interacciones como factores de variación. Las diferencias significativas entre los promedios se determinaron por medio de la prueba de Fisher $(\mathrm{p} \leq 0.05)$.

\section{Análisis instrumental de textura}

La textura instrumental se evaluó utilizando un analizador de textura TA.XT2i (Stable Micro Systems, UK) (Figura 1). 


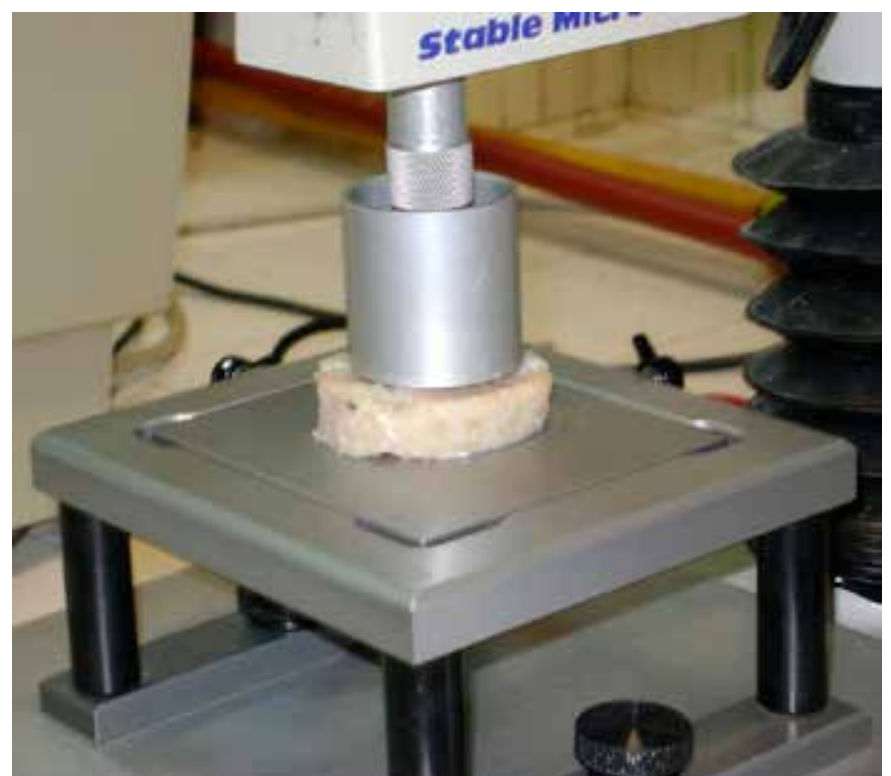

Figura 1. Análisis instrumental de textura con TA.XT2i

Se realizaron ensayos de TPA (Análisis de Perfil de Textura), ejerciendo una doble compresión sobre las muestras con una sonda cilíndrica de $35 \mathrm{~mm}$ de diámetro. Los parámetros seleccionados para el análisis fueron: velocidad de ensayo: $1 \mathrm{~mm} / \mathrm{s}$, deformación de la muestra 40\%, tiempo entre dos compresiones $5 \mathrm{~s}$, célula de carga de $25 \mathrm{~kg}$ (Bourne, 2002). Se registró fuerza ejercida para comprimir la muestra vs. tiempo mediante el software Texture Expert (Versión 1.0, Stable Micro Systems Ltd.). De las curvas de fuerza en función del tiempo se calcularon los siguientes parámetros para caracterizar la textura de las hamburguesas (Bourne, 2002; Kilcast, 2004):

Dureza: pico máximo de fuerza durante la primera compresión.

Elasticidad: relación entre el tiempo en el que se alcanza la fuerza máxima en la segunda y en la primera compresión.

Cohesividad: cociente entre las áreas bajo la curva de la segunda y primera compresión.

Masticabilidad: producto de la dureza, cohesividad y elasticidad.

\section{Análisis estadístico}

Sobre los datos obtenidos se realizó un análisis de varianza (ANOVA), utilizando "muestra" como factor de variación. Las diferencias significativas entre los promedios se determinaron por medio de la prueba de Fisher $(\mathrm{p} \leq 0.05)$.

\section{Resultados}

En las Figuras 2, 3 y 4 se observan las muestras de hamburguesas sin cocción utilizadas para el estudio.

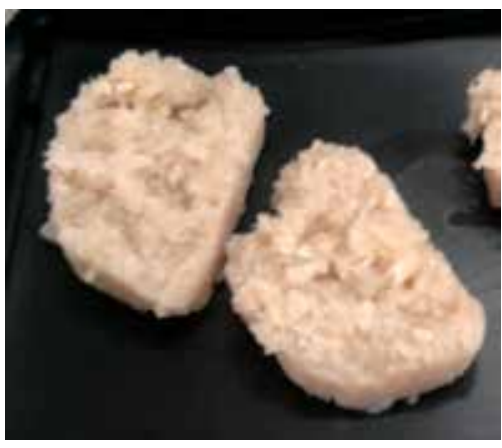

Figura 2. Hamburguesas sin enzima.

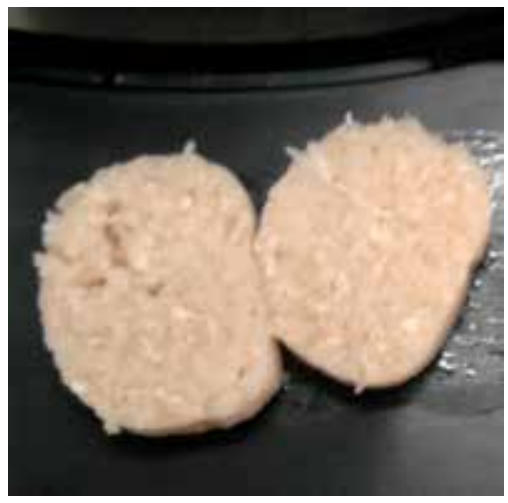

Figura 3. Hamburguesas con $0,5 \%$ de enzima.

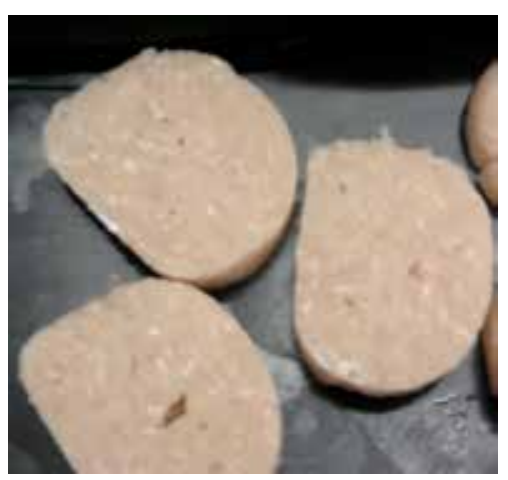

Figura 4. Hamburguesas con $1 \%$ de enzima.

Al retirar las muestras del envoltorio plástico se observó un remanente de líquido en los envases, abundante para la muestra que no contiene enzima, menor para la muestra con $0,5 \%$ de enzima y nulo para la de $1,0 \%$ de enzima. Como se puede observar en las Figuras 2, 3 y 4, las hamburguesas que no tenían enzima en su formulación se desgranaron parcialmente al corte; las hamburguesas con $0,5 \%$ de enzima en su formulación no se desgranaron pero tampoco quedaron muy compactas mientras que las hamburguesas con $1 \%$ de enzima quedaron compactas y firmes al corte.

Los resultados de la evaluación sensorial y del análisis de perfil de textura (TPA) se muestran en las Tablas 2 y 3 . 


\begin{tabular}{|c|c|c|c|}
\hline $\begin{array}{c}\text { Atributos } \\
\text { sensoriales }\end{array}$ & $\begin{array}{c}\text { Hamburguesa } \\
\text { sin enzima }\end{array}$ & $\begin{array}{c}\text { Hamburguesa } \\
\text { con 0,5\% de TG }\end{array}$ & $\begin{array}{c}\text { Hamburguesa } \\
\text { con 1\% de TG }\end{array}$ \\
\hline $\begin{array}{c}\text { Forma (desunifor- } \\
\text { me-uniforme) }\end{array}$ & $7,0^{\mathrm{a}}$ & $5,1^{\mathrm{b}}$ & $1,8^{\mathrm{c}}$ \\
\hline $\begin{array}{c}\text { Aspecto (liso- } \\
\text { granulado) }\end{array}$ & $7,6^{\mathrm{a}}$ & $5,0^{\mathrm{b}}$ & $1,5^{\mathrm{c}}$ \\
\hline $\begin{array}{c}\text { Color (homogéneo- } \\
\text { heterogéneo) }\end{array}$ & $5,9^{\mathrm{a}}$ & $6,1^{\mathrm{a}}$ & $1,2^{\mathrm{b}}$ \\
\hline $\begin{array}{c}\text { Dureza (poco- } \\
\text { mucho) }\end{array}$ & $5,6^{\mathrm{a}}$ & $2,9^{\mathrm{b}}$ & $1,0^{\mathrm{c}}$ \\
\hline $\begin{array}{c}\text { Masticabilidad } \\
\text { (poco-mucho) }\end{array}$ & $7,3^{\mathrm{a}}$ & $5,3^{\mathrm{b}}$ & $2,2^{\mathrm{c}}$ \\
\hline $\begin{array}{c}\text { Humedad bucal } \\
\text { (seco-húmedo) }\end{array}$ & $2,1^{\mathrm{a}}$ & $4,2^{\mathrm{b}}$ & $7,2^{\mathrm{c}}$ \\
\hline $\begin{array}{c}\text { Sabor salado } \\
\text { (nada-mucho) }\end{array}$ & $0,8^{\mathrm{a}}$ & $3,6^{\mathrm{b}}$ & $5,8^{\mathrm{c}}$ \\
\hline $\begin{array}{c}\text { Intensidad de sabor } \\
\text { (poco-mucho) }\end{array}$ & $1,2^{\mathrm{a}}$ & $4,3^{\mathrm{b}}$ & $6,9^{\mathrm{c}}$ \\
\hline
\end{tabular}

Tabla 2. Resultados de la evaluación sensorial.

Letras distintas dentro de una misma fila indican diferencia significativa según prueba de Fisher $(p \leq 0.05)$.

\begin{tabular}{|c|c|c|c|}
\hline $\begin{array}{c}\text { Parámetro de } \\
\text { textura }\end{array}$ & $\begin{array}{c}\text { Hamburguesa } \\
\text { sin enzima }\end{array}$ & $\begin{array}{c}\text { Hamburguesa } \\
\text { con 0,5\% de TG }\end{array}$ & $\begin{array}{c}\text { Hamburguesa } \\
\text { con 1\% de TG }\end{array}$ \\
\hline Dureza & $68,3^{\mathrm{a}}$ & $52,2^{\mathrm{b}}$ & $47,7^{\mathrm{c}}$ \\
\hline Elasticidad & $0,89^{\mathrm{a}, \mathrm{b}}$ & $0,89^{\mathrm{a}}$ & $0,91^{\mathrm{b}}$ \\
\hline Cohesividad & $0,63^{\mathrm{a}}$ & $0,67^{\mathrm{b}}$ & $0,63^{\mathrm{a}}$ \\
\hline Masticabilidad & $38,4^{\mathrm{a}}$ & $31,0^{\mathrm{b}}$ & $27,3^{\mathrm{c}}$ \\
\hline
\end{tabular}

Tabla 3. Parámetros de textura.

Letras distintas dentro de una misma fila indican diferencia significativa según prueba de Fisher $(p \leq 0.05)$.

La mayor parte de los atributos sensoriales y los parámetros de textura se modificaron en forma significativa $(\mathrm{p}<0.05)$ por la incorporación de la TG.

El agregado de TG disminuyó en forma significativa la desuniformidad de la forma, la granulosidad del aspecto, la dureza y la masticabilidad, y aumentó en forma significativa la humedad bucal, el sabor salado y la intensidad del sabor. El único atributo que no se modificó en forma significativa con el agregado de $0,5 \%$ de TG fue la heterogeneidad del color; para lograr que fuera más homogéneo se necesitó un $1,0 \%$ de TG.

De los parámetros de textura, solo la dureza y la masticabilidad disminuyeron en forma significativa al agregar $0,5 \%$ y $1,0 \%$ de TG. La elasticidad y la cohesividad de las muestras con $1,0 \%$ de TG no mostró diferencias respecto a la muestra sin TG.

\section{Discusión}

La formulación de hamburguesas con transglutaminasa ha mostrado un efecto positivo desde el punto de vista sensorial. Visualmente, la hamburguesa con TG se presenta como más homogénea y compacta, con un aspecto más liso y un color más homogéneo, lo que resulta más atractivo para el consumo. En efecto, la transglutaminasa microbiana presenta características de adhesividad de las proteínas componentes de las partículas musculares de la merluza, que hacen y posibilitan la homogeneidad y cohesión de dichas estructuras, las cuales normalmente, por tratarse de fibras musculares cortas, se disgregan.

La dureza y la masticabilidad medidas con el analizador de textura disminuyeron con el agregado de TG, coincidiendo con los resultados de la evaluación sensorial. Esto es muy importante para el paladar del consumidor. En ambos análisis se demostró que el agregado de enzima, en cualquiera de sus dos concentraciones, provoca que sea menor la fuerza necesaria para comprimir la muestra entre los dientes y poder masticarla.

Otro atributo de textura apreciado por los consumidores es la humedad bucal, la cual se observa que se incrementa en gran medida con el agregado de enzima, que aumenta la retención de agua. La gelificación de las proteínas se facilita debido a la función de la transglutaminasa de unir proteínas a través de enlaces inter o intramoleculares, por lo que la capacidad de retención de agua se ve favorecida. La capacidad de retención de agua de la enzima se evidenció también al abrir los envases plásticos de las muestras, y se observó que las hamburguesas con $1,0 \%$ de TG no presentaban líquido remanente.

Estos resultados coinciden con los encontrados por diversos autores que han estudiado el empleo de TG en preparaciones de surimi de varias especies de pescado, que indicaron una mejora en la fuerza del gel (Jiang et al., 2000; Lee et al., 1997; Ramírez et al., 2000; Tsai et al., 1996).

Respecto a los atributos de sabor, existió una diferencia significativa en el sabor salado de las tres muestras y la más salada fue la muestra con mayor concentración de enzima, dado que su formulación incluye cloruro de sodio y fosfato trisódico. De forma similar ocurrió con el atributo intensidad de sabor: la muestra con mayor concentración de enzima presentó mayor intensidad de sabor debido a los componentes que la acompañan.

\section{Conclusiones}

El agregado de transglutaminasa al desmenuzado de merluza para la obtención de hamburguesas se presenta como una posibilidad innovadora y práctica. Genera productos con mejor forma, aspecto y color, menos duros, gomosos y masticables, más húmedos y con sabor más intenso, lo cual los hace atractivos para el público de Uruguay, poco consumidor de productos pesqueros.

El desmenuzado de merluza en la actualidad se vende como commodity. Si se logra introducir en el mercado uruguayo las hamburguesas que se desarrollaron en el marco de este trabajo, se aumentaría su valor agregado al ocupar mano de obra nacional.

Esta investigación busca contribuir a profundizar el conocimiento de la utilización de transglutaminasa de origen microbiano en una especie de pescado uruguaya. A su vez, se plantea la realización de ensayos similares a los aquí presentados en otras especies de pescado, incluyendo aquellas no explotadas como congrio (Conger orbignyanus), sargo (Diplodus argenteus) y pez sable (Trichiurus lepturus), entre otras.

\section{Referencias}

- AGUILAR-ZÁrATE, P.; AGUILAR-ZÁRATE, M.; CARRILLO, I.; PORTILLA, O. Importancia de la producción de transglutaminasa microbiana para su aplicación en alimentos. En: Revista Científica de la Universidad Autónoma de Coahuila. 2012, 4(8):1-17.

- ASAGAMI, T.; OGIWARA, M.; WAKAMEDA, A.; NOGUCHI, S.F. Effect of microbial transglutaminase on the quality of frozen surimi made from various kinds of fish species. En: Fish Sci. 1995, 
61(2):267-272.

- BOURNE, M. Food texture and viscosity: concept and measurement. $2^{\mathrm{a}}$ ed. California: Academic Press, 2002.

- DONDERO, M.; CUROTTO, E.; FIGUEROA, V. Transglutaminase effects on gelation of jack mackerel surimi Trachurus murphyi. En: Food Sci Tech Int. 2002, 8(1):49-54.

- GILLELAND, G.M.; LANIER, T.C.; HAMANN, D.D. Covalent bonding in pressure induced fish protein gels. En: J Food Sci. 1997, 62(4):713-716.

- INTERNATIONAL ORGANIZATION FOR STANDARIZATION (Suiza). ISO 8586-1: Sensory analysis - general guidance for the selection, training, and monitoring of assessors. Part 1-Selected assessors. Ginebra: ISO, 1993.

- INTERNATIONAL ORGANIZATION FOR STANDARIZATION (Suiza). ISO 8589: Sensory analysis: general guidance for the design of test rooms. Ginebra: ISO,1988.

- JIANG, S.T.; HSIEH, F.; HO, M.L.; CHUNG, Y.C. Combination effects of microbial transglutaminase, reducing agent, and protease inhibitor on the quality of hairtail surimi. En: J. Food Sci. 2000, 65: 241-245.

- KILCAST, D. Texture in food. Volume 2: Solid foods. Cambridge: Woodhead Publishing, 2004.

- LEE, H.G.; LANIER, T.C.; HAMANN, D.D.; KNOPP, J.A. Transglutaminase effects on low temperature gelation of fish protein sols. En: J. Food Sci. 1997, 62:20-24.

- LÓPEZ, J. El mercado de pescado en Montevideo. Montevideo: INFOPESCA, 2007.

- MÁRQUEZ, E.; ARÉVALO, E.; BARBOZA, Y.; BENÍTEZ, B.; RANGEL, L.; ARCHILE, A. Efecto de la concentración de transglutaminasa y tiempo de reacción en la estabilidad de productos reestructurados. En: Revista Cientifica, FCV-LUZ. 2006,16(6):662-667.

- MAZZA PÉREZ, C. A. Mejoramiento de los mercados internos de productos pesqueros en América Latina y el Caribe. Montevideo: DINARA, 2000.

- RAMÍREZ, J.A.; SANTOS, I.A.; MORALES, O.G.; MORRISSEY, M.T.; VÁZQUEZ, M. Application of microbial transglutaminase to improve mechanical properties of surimi from silver carp. En: Cienc. Tecnol. Aliment. 2000, 3:21-28.

- SAKAMOTO, H.; KUMASAWA, Y.; MOTOKI, M. Strength of protein gels prepared with microbial transglutaminase as related to reaction conditions. En: Journal of Food Science. 1994, 59(4):866-871.

- STEFFOLANI, M. E. Efecto de las enzimas pentosanasa, glucosa oxidasa y transglutaminasa en productos de panificación. La Plata: Universidad Nacional de La Plata, 2012.

- TSAI, G.J.; LIN, S.M.; JIANG, S.T. Transglutaminase from Streptoverticillium ladakanum and application to minced fish product. En: J. Food Sci. 1996, 61:1234-1238. 